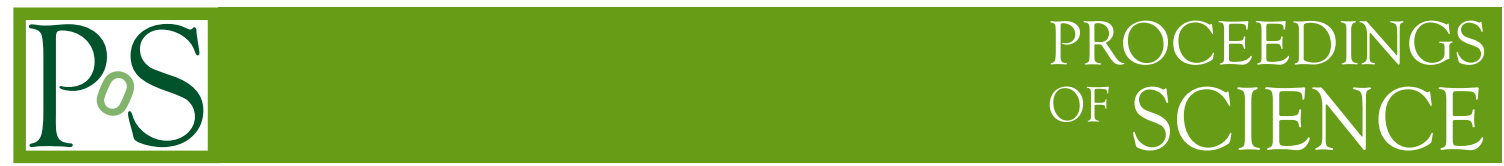

\title{
QCD with Flavored Minimally Doubled Fermions
}

\author{
Johannes Heinrich Weber * \\ Technische Universität München, Physik Department T30f, James-Franck-Str. 1, 85748 \\ Garching, Germany \\ E-mail: johannes.weber@tum. de
}

I discuss minimally doubled fermions fermions as an ultra-local formulation on the lattice for sea quarks that realize a non-singlet chiral symmetry. I introduce a non-singlet mass term for Karsten-Wilczek fermions and identify the appropriate representation of the SU(2) flavor group at finite lattice spacing. I present an algebraic proof that the symmetry of the quark determinant under charge conjugation and reflections of the Euclidean axes is preserved for Karsten-Wilczek fermions as sea quarks. Finally, I discuss how the flavor components in meson correlation functions with Karsten-Wilczek fermions emerge naturally and I show how taste-breaking can be avoided without fine tuning.

34th annual International Symposium on Lattice Field Theory

24-30 July 2016

University of Southampton, UK

\footnotetext{
* Speaker.
} 


\section{Introduction}

Since the early years of lattice field theory the realization of chiral symmetry has always been an intricate topic. Minimally doubled fermions (MDF) are one of the few approaches that realize an exact chiral symmetry on the lattice. Hence, with MDF one may study light or even massless quarks. MDF are ultra-local and their Dirac operators are sparse matrices, so they are numerically faster than Ginsparg-Wilson fermions. Due to having a single pair of fermions, MDF have been considered for representing a degenerate light quark doublet. However, already the earliest studies suggested that hypercubic symmetry must be broken for MDF, giving rise to anisotropy, and thus, requiring extra counterterms. Karsten's initial suggestion, its further refinement by Wilzcek, and the study of its broken charge conjugation and reflection symmetries by Pernici are the published works [1] of the early era before the field fell dormant for a decade. The field was reinvigorated by Boriçi and Creutz in 2007, cf. Refs. [2], with many new contributions appearing in a brief time span thereafter. New patterns for attaining minimal doubling had been suggested though none of these made it beyond an exploratory stage. Only a few variants of MDF have been studied systematically with respect to their use in QCD. In particular, the symmetries of Karsten-Wilczek fermions (KWF) have been scrutinized again [3], their counterterms have been calculated perturbatively [4], and the axial anomaly has been identified [5].

Numerical studies of KWF were published in Refs. [6], where two independent non-perturbative methods for tuning the relevant counterterm have been identified, one based on the anisotropy of hadronic observables, the other based on a Fourier analysis of the changed pole structure of the quark propagators. It has been shown that the broken time reflection symmetry is usually not observed in meson correlation functions and an analytical proof has been presented. For KWF, Goldstone boson-like behavior of the pseudoscalar ground state at finite lattice spacing, the appearance of parity partner states, and taste breaking have been observed. Two higher-dimension operators necessary for the Symanzik improvement program have also been identified. However, previous numerical studies of MDF were performed in the quenched approximation. The chief worry [6] regarding dynamical MDF has been that it seems possible that the charge conjugation and reflection symmetries might be broken for the gauge configurations as well. In the following, I will show that this concern is unfounded. In Section 2, I will introduce a non-singlet mass term for controlled flavor symmetry breaking and identify the appropriate representation of the flavor $\mathrm{SU}(2)$ group on the lattice. In Section 3, I present a proof that the vacuum does not suffer from the broken discrete symmetries. In Section 4, I outline the strategies for identifying flavored mesons and discuss how taste-breaking arises, and how it can be avoided.

\section{Karsten-Wilczek fermions and flavor}

At tree level, degenerate KWF are realized with the Dirac operator

$$
\mathscr{D}=D+m_{0}+[3 r K+r W], \quad K=\frac{i}{a_{\tau}} \gamma_{0}, \quad W=-\frac{i}{a_{\tau}} \gamma_{0}\left(3+a_{\sigma}^{2} \boldsymbol{D} \cdot \boldsymbol{D}^{*}\right),
$$

where $D=\sum_{\mu} \gamma_{\mu} D_{\mu}$ is the naïve Dirac operator. Together $K$ and $W$ form the Karsten-Wilczek term. The free propagator has only two poles at $k_{0}=0$ or $\pi / a$ (with $k=0$ ) if $r^{2}>1 / 4$. The 
fermion action is invariant under the cubic group $\left(W_{3}\right)$, parity $(\mathscr{P})$, and joint application of charge conjugation $(\mathscr{C})$ and time reflection $(\mathscr{T})$, Ref. [3]. Furthermore, $\mathscr{D}$ satisfies $\gamma_{5}$ hermiticity.

Three counterterms are required in the interacting theory. The operators $c_{3} K$ and $d_{4} \gamma_{0} D_{0}$ serve as fermionic counterterms. In perturbation theory, $c_{3}$ is known to be an odd function of $r$. In the following, I incorporate the dimension four counterterm $d_{4} \gamma_{0} D_{0}$ in the operator $D$, since it transforms in exactly the same way as long as no rotations beyond the cubic group $W_{3}$ are considered. Furthermore, I incorporate the coefficient $c_{3}$ in $\rho=3 r+c_{3}$. The gluonic counterterm can be written in terms of temporal plaquette operators, e.g. the Wilson gauge action with counterterm reads

$$
S^{g}[U]=\sum_{n} \beta_{\sigma} \sum_{j<k} P_{n}^{j k}+\beta_{\tau}\left(1+d_{p}\right) \sum_{j} P_{n}^{j 0},
$$

where $P_{m}^{\mu v}=\operatorname{Re} \operatorname{tr}\left(1-U_{m}^{\mu v}\right) / N_{c}$ is the normalized trace of the real part of the plaquette operator subtracted from its constant part. For the isotropic Wilson gauge action, the counterterms have been calculated at 1-loop level [4] and $c_{3}$ even has been determined non-perturbatively [6]. The global symmetries of the bare and the renormalized action are the same at finite lattice spacing.

A non-singlet mass term can be realized in the form ${ }^{1}$ of

$$
m_{3} M, \quad M=\left(1+a_{\tau}^{2} D_{0} D_{0}^{*}\right)\left(3+a_{\sigma}^{2} \boldsymbol{D} \cdot \boldsymbol{D}^{*}\right),
$$

which leaves all of the previously mentioned symmetries intact. Of course, the chiral transform $\left(\chi=e^{i \gamma_{5} \alpha}\right)$ is a symmetry of the action only if both masses vanish $\left(m_{0}=m_{3}=0\right)$. Nevertheless, there is another symmetry that is broken only by including the non-singlet mass term, Eq. (2.3), in the Dirac operator, Eq. (2.1). This is the so-called mirror fermion symmetry, which has been discovered by Pernici but generally been ignored. The mirror fermion symmetry arises because the action is invariant under joint application of time reflection or charge conjugation and a temporal shift transform $(\tau)$. Here, I define the shift transform in terms of the self-inverse operator $\tau=$ $\tau_{0}(-1)^{n_{0}}$, where $\tau_{0}=i \gamma_{0} \gamma_{5}$. While $M$ is invariant under $\mathscr{C}$ and $\mathscr{T}$, as required for a scalar mass operator, $M$ changes sign under $\tau$. Thus, $\tau$ is a representative of some SU(2) flavor matrix. The appropriate (self-inverse) representation of the full set of $\mathrm{SU}(2)$ flavor matrices is given by

$$
\left(\begin{array}{c}
\lambda \\
\tau \\
\vartheta
\end{array}\right)=\left(\begin{array}{c}
\gamma_{0}(-1)^{n_{\sigma}} \\
i \gamma_{0} \gamma_{5}(-1)^{n_{0}} \\
\gamma_{5}(-1)^{\bar{n}}
\end{array}\right)=\left(\begin{array}{c}
\sigma_{1} \times(-1)^{n_{\sigma}} \\
\sigma_{2} \times(-1)^{n_{0}} \\
\sigma_{3} \times(-1)^{\bar{n}}
\end{array}\right) \otimes \mathbf{1}_{2 \times 2},
$$

where $n_{\sigma}=\sum_{j} n_{j}$ and $\bar{n}=\sum_{\mu} n_{\mu} \cdot\{\lambda, \tau, \vartheta\}$ trivially satisfy the $\mathrm{SU}(2)$ algebra.

From these considerations, I derive the full symmetry pattern of the Dirac operator $\mathscr{D}_{\kappa}=\mathscr{D}+m_{3} M$ and summarize it in Table 1. Due to the SU(2) algebra and the $\mathscr{C} \mathscr{P} \mathscr{T}$ invariance, out of each triplet (boxes in the table) only two transforms are independent. Hence, a set of five independent unitary transforms remains and each term in the Dirac operator $\mathscr{D}_{\mathcal{K}}$ transforms differently under the set.

\footnotetext{
${ }^{1}$ There are various other possibilities to realize a non-singlet mass term, but unless a $W_{3}$ symmetric spatial hopping term is included, they will lead to an unfavorable flavor structure.
} 


\begin{tabular}{|c|c|c|c|c|c|c|c|}
\hline $\mathscr{O}$ & $D$ & $K$ & $W$ & $\mathbf{1}$ & $M$ & $U^{0}$ & $U^{j}$ \\
\hline$\chi$ & $+D$ & $+K$ & $+W$ & $-\mathbf{1}$ & $-M$ & $U^{0}$ & $U^{j}$ \\
\hline \hline$\lambda$ & $+D$ & $+K$ & $-W$ & $+\mathbf{1}$ & $-M$ & $U^{0}$ & $U^{j}$ \\
$\tau$ & $+D$ & $-K$ & $-W$ & $+\mathbf{1}$ & $-M$ & $U^{0}$ & $U^{j}$ \\
$\vartheta$ & $+D$ & $-K$ & $+W$ & $+\mathbf{1}$ & $+M$ & $U^{0}$ & $U^{j}$ \\
\hline \hline $\mathscr{P}$ & $+D$ & $+K$ & $+W$ & $+\mathbf{1}$ & $+M$ & $+U^{0}$ & $-U^{j \dagger}$ \\
$\mathscr{T}$ & $+D$ & $-K$ & $-W$ & $+\mathbf{1}$ & $+M$ & $-U^{0 \dagger}$ & $U^{j}$ \\
$\mathscr{C}$ & $+D^{T}$ & $-K^{T}$ & $-W^{T}$ & $+\mathbf{1}^{\mathbf{T}}$ & $+M^{T}$ & $+U^{0 *}$ & $U^{j *}$ \\
\hline
\end{tabular}

Table 1: Symmetry pattern of the Dirac operator $\mathscr{D}_{\kappa} \cdot d_{4} \gamma_{0} D_{0}$ transforms like $D$ and is incorporated there.

Only a single operator, namely $\mathscr{P}$, leaves each term invariant. Note that there is no unambiguous map between $\{\lambda, \tau, \vartheta\}$ and the three $\mathrm{SU}(2)$ generators. However, in order to interpret $m_{3} M$ in Eq. (2.3) as a flavor-diagonal mass term, the assignment to the generators should be as in the explicit $2 \times 2$ notation in Eq. (2.4): $\vartheta$ corresponds to $\sigma_{3}$. This suggests associating left-handed quarks with one flavor and right-handed quarks with the other. Moreover, to avoid quarks with negative mass, the renormalized singlet and non-singlet masses must satisfy $m_{0}^{R} \geq m_{3}^{R}$.

\section{Karsten-Wilczek fermions as sea quarks}

With KWF as sea quarks, the quark determinant is calculated from an operator $\mathscr{D}_{\kappa}$ that breaks four out of five independent discrete symmetries. In the following, I prove that the determinant nevertheless preserves all these symmetries. This outcome is analogous to twisted mass Wilson fermions, where the twist term breaks $\mathscr{P}$ while the vacuum is invariant under $\mathscr{P}$.

The key strategy is to introduce products of signs $s, x, y, z= \pm 1$ in front of all operators that transform non-trivially under the broken symmetries, such that every sign corresponds to one of the four broken symmetries. Then no operator that depends explicitly on any of these signs is invariant, but any operator that is independent of all signs is invariant under all broken symmetries. With assignments $\mathrm{s} \leftrightarrow \mathscr{C}, \mathrm{x} \leftrightarrow \chi, \mathrm{y} \leftrightarrow \lambda$ and $\mathrm{z} \leftrightarrow \vartheta$ I rewrite the full Dirac operator ${ }^{2}$ as

$$
\mathscr{D}_{\kappa_{\mathrm{s}, \mathrm{x}, \mathrm{z}, \mathrm{z}}}=D+\mathrm{x} m_{0}+\mathrm{sz} \rho K+\mathrm{sy} r W+\mathrm{xy}_{3} M
$$

and find the eigenvalue equation for eigenvalue $\omega_{\kappa_{s, x, y, z}}$ and eigenvectors $\phi_{\kappa_{s, x, y, z}}^{\omega}$ to be

$$
\bar{\phi}_{\kappa_{s, x, y, z}}^{\omega} \mathscr{D}_{\kappa_{s, x, y, z}} \phi_{\kappa_{s, x, y, z}}^{\omega}=\omega_{\kappa_{s, x, y, z}} .
$$

Next, I prove that $\operatorname{det} \mathscr{D}_{\kappa_{s, x, y, z}}$ is not affected by the broken discrete symmetries as it is independent of the signs s, x,y,z and hence satisfies $\operatorname{det} \mathscr{D}_{\kappa} \equiv \operatorname{det} \mathscr{D}_{\kappa_{\mathrm{s}, \mathrm{x}, \mathrm{z}, \mathrm{z}}}$. In the following, I omit s, x, y, z unless I specifically discuss the dependence on a sign and attach it to the multi-index $\kappa$, e.g. $\kappa_{+x}$ or $\kappa_{-\times}$.

0) If there are massless quarks (due to $m_{0}=m_{3}=0$ or due to cancellation between the two mass terms), then there is at least one exact zero mode and the determinant vanishes as it is the

\footnotetext{
${ }^{2} \kappa=\left(m_{0}, m_{3}, r, c_{3}, d_{4} ; d_{p}, \beta_{\sigma}, \beta_{\tau}\right)$ is to be understood as a multi-index of bare parameters and counterterms. I use an extended multi-index $\kappa_{\mathrm{s}, \mathrm{x}, \mathrm{y}, \mathrm{z}}=(\kappa, \mathrm{s}, \mathrm{x}, \mathrm{y}, \mathrm{z})$, since the role of $\mathrm{s}, \mathrm{x}, \mathrm{y}, \mathrm{z}$ is different from the 'physical' parameters of $\kappa$.
} 
product of the eigenvalues of the Dirac operator, $\operatorname{det} \mathscr{D}_{\kappa}=\prod_{\omega_{\kappa}} \omega_{\kappa}$. Thus, det $\mathscr{D}_{\kappa}$ (being zero) would be trivially independent of all signs ${ }^{3}$. Hence, I exclude zero mass in the following.

1) First, I use $\gamma_{5}$ hermiticity of $\mathscr{D}_{\kappa}$. It follows that eigenvalues form complex conjugate pairs, so if $\omega_{\kappa}$ is an eigenvalue, so is $\omega_{\kappa}^{*}$. Thus, $\operatorname{det} \mathscr{D}_{\kappa}$ is real and strictly positive (since quark mass zero has been excluded). Next, I combine the positivity with chiral symmetry and obtain

$$
0<\operatorname{det} \mathscr{D}_{\kappa_{+\times}}=\sqrt{\left(\operatorname{det} \mathscr{D}_{\kappa_{+\times}} \operatorname{det}\left[\gamma_{5} \mathscr{D}_{\kappa_{+\times}} \gamma_{5}\right]\right)}=\sqrt{\left(\operatorname{det} \mathscr{D}_{\kappa_{+\times}} \operatorname{det}\left[-\mathscr{D}_{\kappa_{-\times}}\right]\right)} .
$$

Since quark masses are non-zero, the mass matrix $m_{0}+m_{3} M$ is invertible and I find

$$
0<\operatorname{det} \mathscr{D}_{\kappa_{+\times}}=\sqrt{\left(\operatorname{det}\left(m_{0}+m_{3} M\right)^{2} \operatorname{det}\left[1-\left(\frac{D+\rho K+r W}{m_{0}+m_{3} M}\right)^{2}\right]\right)},
$$

which is clearly independent of $x$. In fact, even $\left|\omega_{\kappa}\right| \equiv\left|\omega_{\kappa_{+\times}}\right|=\left|\omega_{\kappa_{-x}}\right|$ is satisfied by each eigenvalue and $\operatorname{det} \mathscr{D}_{\kappa}$ can be written as $\operatorname{det} \mathscr{D}_{\kappa_{\mathrm{x}}}=\prod_{\omega_{\kappa \mathrm{x}}} \omega_{\kappa_{\mathrm{x}}}=\prod_{\omega_{\kappa_{\mathrm{x}}}}\left|\omega_{\kappa_{\mathrm{x}}}\right|=\prod_{\omega_{\kappa}}\left|\omega_{\kappa}\right|$.

2) Second, I plug the SU(2) generators $\lambda$ or $\vartheta$ into the eigenvalue equation, Eq. (3.2). For $\lambda$, I define $\varphi_{\kappa_{-y}}^{\omega}=\lambda \phi_{\kappa_{+y}}^{\omega}$ and $\bar{\varphi}_{\kappa_{-y}}^{\omega}=\bar{\phi}_{\kappa_{+y}}^{\omega} \lambda$ and find an equality of eigenvalues,

$$
\omega_{\kappa_{+y}}=\bar{\phi}_{\kappa_{+y}}^{\omega} \mathscr{D}_{\kappa_{+y}} \phi_{\kappa_{+y}}^{\omega}=\bar{\phi}_{\kappa_{+y}}^{\omega} \lambda \lambda \mathscr{D}_{\kappa_{+y}} \lambda \lambda \phi_{\kappa_{+y}}^{\omega}=\bar{\varphi}_{\kappa_{-y}}^{\omega} \mathscr{D}_{\kappa_{-y}} \varphi_{\kappa_{-y}}^{\omega}=\omega_{\kappa_{-y}} .
$$

Hence, $\omega_{\kappa} \equiv \omega_{\kappa_{+y}}=\omega_{\kappa_{-y}}$. Since $y^{2}=1$ this implies that $\omega_{\kappa}$ is independent of y. Using $\vartheta$, I reiterate the same steps and find that $\omega_{\kappa} \equiv \omega_{\kappa_{+z}}=\omega_{\kappa_{-z}}$ is also independent of z.

3) In the third step,I repeat this procedure by plugging the charge conjugation operator $\mathscr{C}$ into Eq. (3.2). I define charge conjugated eigenvectors $\left(\varphi_{\mathcal{K}_{-s}}^{\omega c}\right)^{T}=-\bar{\phi}_{\mathcal{K}_{+\mathrm{s}}}^{\omega} \mathscr{C}$ and $\left(\bar{\varphi}_{\mathcal{K}_{-\mathrm{s}}}^{\omega c}\right)^{T}=\mathscr{C} \phi_{\mathcal{K}_{\mathrm{s}}}^{\omega}$, and find,

$$
\begin{aligned}
& \omega_{\kappa_{+\mathrm{s}}}=\bar{\phi}_{\kappa_{+\mathrm{s}}}^{\omega}[U] \mathscr{D}_{\kappa_{+\mathrm{s}}}[U] \phi_{\kappa_{+\mathrm{s}}}^{\omega}[U]=\bar{\phi}_{\kappa_{+\mathrm{s}}}^{\omega}[U] \mathscr{C} \mathscr{C} \mathscr{D}_{\kappa_{+\mathrm{s}}}[U] \mathscr{C} \mathscr{C} \phi_{\kappa_{+\mathrm{s}}}^{\omega}[U] \\
& =-\left(\varphi_{\mathcal{K}_{-\mathrm{s}}}^{\omega c}[U]\right)^{T}\left(\mathscr{D}_{\mathcal{K}_{-\mathrm{s}}}[U]\right)^{T}\left(\bar{\varphi}_{\mathcal{K}_{-\mathrm{s}}}^{\omega}[U]\right)^{T}=\left(\bar{\phi}_{\mathcal{K}_{-\mathrm{s}}}^{\omega}[U] \mathscr{D}_{\mathcal{K}_{-\mathrm{s}}}[U] \phi_{\mathcal{K}_{-\mathrm{s}}}^{\omega}[U]\right)^{T}=\omega_{\mathcal{K}_{-\mathrm{s}}} .
\end{aligned}
$$

Hence, $\omega_{\kappa} \equiv \omega_{\kappa_{+s}}=\omega_{\kappa_{-s}}$ is independent of s following the previous line of reasoning.

Hence, I find that the moduli of the eigenvalues and the full determinant are independent of the signs, $\left|\omega_{\kappa}\right| \equiv\left|\omega_{\kappa_{s, x, y, z}}\right|$ and $\operatorname{det} \mathscr{D}_{\kappa} \equiv \operatorname{det} \mathscr{D}_{\kappa_{s, x, y, z}}$. Therefore, the quark determinant is not affected by the broken charge conjugation and reflection symmetries. This statement holds non-perturbatively and is independent of any parameters of the theory as well as of the gauge fields. Since the Wilson gauge action with counterterm is invariant under these symmetries, this also holds for the QCD vacuum/medium. I note in passing that field configurations are automatically $\mathscr{O}(a)$ improved and depend on the bare parameters of the quark action only through $m_{0}^{2}, m_{3}^{2}$ and $r^{2}$.

\section{Flavor components in meson correlation functions}

In the following I stress only the dependence on $\mathrm{s}$ and $m_{3}$, which encode taste and flavor structure of meson correlation functions. It is key to introduce charge conjugation and su(2) projectors, i.e.

$$
\widehat{P}_{\mathscr{C}}^{ \pm}=\frac{1}{2}(1 \pm \mathscr{C}), \quad \widehat{P}_{\tau}^{ \pm}=\frac{1}{2}(1 \pm \tau),
$$

\footnotetext{
${ }^{3}$ The arguments 2) and 3), which show that $\omega_{\kappa} \equiv \omega_{\kappa_{\mathrm{s}, y, z}}$ is independent of s, y and z, are independent of the masses.
} 
and decompose $\mathscr{D}_{\kappa_{\mathrm{s}}}$ and the quark propagator $S=D_{\kappa_{\mathrm{s}}}^{-1}$ using the projectors. I find

$$
\begin{array}{lll}
S=S^{E}+S^{O}, & S^{E}=\left(E-O E^{-1} O\right)^{-1}, & S^{O}=-S^{E} O E^{-1}, \\
S=S^{\eta}+\mathrm{s} S^{\omega}, & S^{\eta}=\left(\eta-\omega \eta^{-1} \omega\right)^{-1}, & S^{\omega}=-S^{\eta} \omega \eta^{-1}
\end{array}
$$

where $E=D+m_{0}$ and $O=m_{3} M+\mathrm{s} \rho K+\mathrm{s} r W$ as well as $\eta=D+m_{0}+m_{3} M$ and $\omega=\rho K+r W$ are the even and odd operators under $\widehat{P}_{\tau}^{ \pm}$and $\widehat{P}_{\mathscr{C}}^{ \pm}$. Note that s is taken out from $\omega$ and $S^{\omega}$ explicitly.

Mesons are created and annihilated through quark-bilinear interpolating operators. The kernels $\Gamma$ of such interpolating operators at source $\left(\Gamma_{a}\right)$ and sink $\left(\Gamma_{b}\right)$ can be classified into even and odd matrices under $\widehat{P}_{\mathscr{C}}^{ \pm}$and $\widehat{P}_{\tau}^{ \pm}$, e.g. for on-site operators under $\widehat{P}_{\mathscr{C}}^{ \pm}$the sets are

$$
\left\{\Gamma^{\eta}\right\}=\left\{\mathbf{1}, \gamma_{5}, i \gamma_{\mu} \gamma_{5}\right\}, \quad\left\{\Gamma^{\omega}\right\}=\left\{\gamma_{\mu}, i \gamma_{\mu} \gamma_{v}\right\}
$$

Depending on the kernels used, only a limited set of the propagator components contribute to any given meson correlation function. In fact, for sufficiently symmetric choices (either $\Gamma_{a}, \Gamma_{b} \in\left\{\Gamma^{\eta}\right\}$ or $\left.\Gamma_{a}, \Gamma_{b} \in\left\{\Gamma^{\omega}\right\}\right)$ any terms linear in s that would violate $\mathscr{C}$ and $\mathscr{T}$ automatically vanish. One would exclusively have such unphysical terms for sufficiently antisymmetric $\Gamma_{a}, \Gamma_{b}$. These terms cancel explicitly upon averaging $s= \pm 1$ for the valence quarks. Because the quark sea is the same for $\mathrm{s}= \pm 1$, this average does not introduce any partial quenching. I note in passing that such meson correlation functions and derived observables are automatically $\mathscr{O}(a)$ improved. In the following I always assume sufficiently symmetric $\Gamma_{a}, \Gamma_{b}$.

Next, I nest the projections of the propagator and take out any linear $m_{3}$ dependence,

$$
S=S^{\eta E}+m_{3} S^{\eta O}+\mathrm{s} m_{3} S^{\omega E}+\mathrm{s} S^{\omega O} .
$$

The kernels $\left\{\Gamma^{\eta E}\right\}$ and so on are obtained through an obvious generalization of Eq (4.4). The propagators and interpolator kernels are intertwined in the quark-disconnected contribution,

$$
\begin{aligned}
C_{\Gamma_{a}, \Gamma_{b}}^{\text {disc }}\left(n_{0}\right) & =\left\langle S^{\eta E} \Gamma^{\eta E}\right\rangle\left\langle S^{\eta E} \Gamma^{\eta E}\right\rangle+\left\langle S^{\omega O} \Gamma^{\omega O}\right\rangle\left\langle S^{\omega O} \Gamma^{\omega O}\right\rangle \\
& +\left(\left\langle S^{\eta O} \Gamma^{\eta O}\right\rangle\left\langle S^{\eta O} \Gamma^{\eta O}\right\rangle+\left\langle S^{\omega E} \Gamma^{\omega E}\right\rangle\left\langle S^{\omega E} \Gamma^{\omega E}\right\rangle\right) \times m_{3}^{2},
\end{aligned}
$$

where the $\langle\ldots\rangle$ denotes combined color-spin trace and gauge average. Spatial summation is implied. Here, association of the traces with either source or sink is not relevant. Thus, the quarkdisconnected contribution for $\gamma_{5}=\Gamma_{a, b} \in\left\{\Gamma^{\eta O}\right\}$ starts at $\mathscr{O}\left(m_{3}^{2}\right)$, which unambiguously identifies the lowest-lying (non-vacuum) state as a neutral pion. I also find that $i \gamma_{0} \gamma_{5}=\Gamma_{a, b} \in\left\{\Gamma^{\eta E}\right\}$ starts at $\mathscr{O}(1)$, which identifies the lowest-lying state as the flavor singlet eta.

The propagator and kernels are not intertwined in the quark-connected contribution. By inserting $\mathbf{1}=\tau^{2}$ at the source and commuting one $\tau$ to the sink, the parity partners are made explicit,

$$
\begin{aligned}
C_{\Gamma_{a}, \Gamma_{b}}^{\mathrm{con}}\left(n_{0}\right) & =\left\langle S^{E} \tau^{2} \Gamma_{a} S^{E} \Gamma_{b}\right\rangle+\left\langle S^{O} \tau^{2} \Gamma_{a} S^{O} \Gamma_{b}\right\rangle=\left\langle S^{E} \tau \Gamma_{a} S^{E} \Gamma_{b} \tau\right\rangle-\left\langle S^{O} \tau \Gamma_{a} S^{O} \Gamma_{b} \tau\right\rangle \\
& =\left(\left\langle S^{E} \tau_{0} \Gamma_{a} S^{E} \Gamma_{b} \tau_{0}\right\rangle-\left\langle S^{O} \tau_{0} \Gamma_{a} S^{O} \Gamma_{b} \tau_{0}\right\rangle\right) \times(-1)^{n_{0}} .
\end{aligned}
$$

Hence, the kernel $\Gamma$ also overlaps with states that would be naïvely associated with a kernel $\Gamma \tau_{0}$. These extra states have opposite parity, which can be understood from the explicit form of $\tau_{0}$. 
The extra states also have $m_{3}^{2} \rightarrow-m_{3}^{2}$ and change the sign of terms with two instances of $S^{O}$, which becomes clear by inserting the propagator with nested projections, Eq. (4.5). Thus, these extra states are composed of unlike quark flavors in a taste-breaking combination. By either setting $s \rightarrow$ $-\mathrm{s}$ or $m_{3} \rightarrow-m_{3}$ on one of the two quark propagators, states with unlike quark flavors but without taste-breaking are obtained (for $\mathrm{s} \rightarrow-\mathrm{s}$ as extra states, for $m_{3} \rightarrow-m_{3}$ as conventional states). Note that there cannot be any quark-disconnected contribution to the extra states at all. Thus, $\gamma_{0}=\Gamma_{a, b} \in\left\{\Gamma^{\omega O}\right\}$ yields exclusively charged pseudoscalars as extra states in the quark-connected contribution, since no quark-antiquark states with $J^{P C}=0^{+-}$are realized in QCD. The quarkconnected contribution for $\mathbf{1}=\Gamma_{a, b} \in\left\{\Gamma^{\eta E}\right\}$ also contains charged pseudoscalars as extra states, though it also contains the conventional scalar with a quark-disconnected contribution starting at $\mathscr{O}(1)$. These extra ground states are genuine charged pions due to the non-existence of a quarkdisconnected contribution. Lastly, there are scalar extra states for $i \gamma_{0} \gamma_{5}=\Gamma_{a, b} \in\left\{\Gamma^{\eta E}\right\}$, which must be interpreted as charged scalars and are actually unphysical.

\section{Conclusions}

In these proceedings I have discussed QCD with flavored minimally doubled fermions. With a nonsinglet mass term there is a natural representation of the $S U(2)$ flavor group at finite lattice spacing. I have presented a proof that the quark determinant and thus also the QCD vacuum/medium are protected from the explicitly broken charge conjugation and reflection symmetries. Flavored mesons can be identified easily by means of appropriate projection operators and are indeed distinguishable through their quark mass dependence and their quark-disconnected contributions. Taste-breaking can be removed without need of fine tuning. Appropriately defined correlation functions are protected from the broken time reflection symmetry. Field configurations as well as appropriately defined meson correlation functions are automatically $\mathscr{O}(a)$ improved and the quark mass dependence is quadratic as it befits chiral fermions. A more comprehensive and more extended discussion of QCD with flavored MDF is in preparation. With this evidence in place, it is about time to start using minimally doubled fermions as sea quarks in lattice QCD.

\section{References}

[1] L. Karsten - Phys. LetT. B 104, 315 (1981);

F. WILCZEK - PRL 59 (1987) 2397;

M. Pernici - Phys. Lett. B346 (1995) 99.

[2] M. CreutZ - JHEP 04 (2008) 017, PoS Lattice2008 080;

A. BoriçI - Phys. Rev. D 78 (2008) 074504, PoS LatTICE2008 231.

[3] P.F. Bedaque, M.I. Buchoff, B.C. Tiburzi, A. Walker-Loud - Phys. Lett. B662 (2008), Phys. Rev. D78; PoS LATTICE2008 068.

[4] S. Capitani, M. Creutz, J.H.W., H. Wittig - JHEP 1009 (2010) 027.

[5] B.C. Tiburzi - Phys. Rev. D82 (2010) 034511.

[6] J.H.W., S. Capitani, H. Wittig - PoS Lattice2013 122;

J.H.W. - POS LATTICE2014 071;

J.H.W. - PhD THESIS: HTTP://UBM.OPUS.HBZ-NRW.DE/VOLLTEXTE/2015/4232/. 